\title{
(6) OPEN ACCESS \\ Effectiveness of a hospital-initiated smoking cessation programme: 2-year health and healthcare outcomes
}

\author{
Kerri A Mullen, ${ }^{1}$ Douglas G Manuel, ${ }^{2}$ Steven J Hawken, ${ }^{2}$ Andrew L Pipe, ${ }^{1}$ \\ Douglas Coyle, ${ }^{3}$ Laura A Hobler, ${ }^{1}$ Jaime Younger, ${ }^{2}$ George A Wells, ${ }^{1}$ Robert D Reid ${ }^{1}$
}

\begin{abstract}
- Additional material is published online only. To view please visit the journal online (http://dx.doi.org/10.1136/ tobaccocontrol-2015-052728).

'University of Ottawa Heart Institute, Ottawa, Ontario, Canada

${ }^{2}$ Ottawa Hospital Research Institute, Ottawa, Ontario, Canada

${ }^{3}$ University of Ottawa, Ottawa, Ontario, Canada
\end{abstract}

Correspondence to

Dr Kerri Anne Mullen, 40 Ruskin Street, H2353, Ottawa, Ontario,

Canada K1Y 4W7;

kmullen@ottawaheart.ca

Received 25 September 2015 Accepted 20 April 2016 Published Online First 17 May 2016

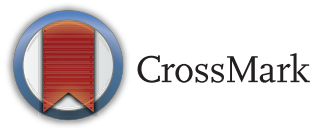

To cite: Mullen KA, Manuel DG, Hawken SJ, et al. Tob Control 2017;26:293-299.

\section{ABSTRACT}

Background Tobacco-related illnesses are leading causes of death and healthcare use. Our objective was to determine whether implementation of a hospitalinitiated smoking cessation intervention would reduce mortality and downstream healthcare usage.

Methods A 2-group effectiveness study was completed comparing patients who received the 'Ottawa Model' for Smoking Cessation intervention ( $n=726)$ to usual care controls ( $n=641$ ). Participants were current smokers, $>17$ years old, and recruited during admission to 1 of 14 participating hospitals in Ontario, Canada. Baseline data were linked to healthcare administrative data.

Competing-risks regression analysis was used to compare outcomes between groups.

Results The intervention group experienced significantly lower rates of all-cause readmissions, smoking-related readmissions, and all-cause emergency department (ED) visits at all time points. The largest absolute risk reductions (ARR) were observed for allcause readmissions at 30 days $(13.3 \%$ vs $7.1 \%$; ARR, $6.1 \%(2.9 \%$ to $9.3 \%) ; p<0.001), 1$ year $(38.4 \%$ vs $26.7 \% ;$ ARR, $11.7 \%(6.7 \%$ to $16.6 \%) ; p<0.001)$, and 2 years $(45.2 \%$ vs $33.6 \%$; ARR, $11.6 \%$ (6.5\% to $16.8 \%) ; p<0.001)$. The greatest reduction in risk of allcause ED visits was at 30 days (20.9\% vs $16.4 \%$; ARR, $4.5 \%(0.4 \%$ to $8.7 \%) ; p=0.03)$. Reduction in mortality was not evident at 30 days, but significant reductions were observed by year $1(11.4 \%$ vs $5.4 \%$; ARR $6.0 \%$ (3.1\% to $9.0 \%) ; p<0.001)$ and year $2(15.1 \%$ vs $7.9 \%$; ARR, $7.3 \%$ (3.9\% to $10.7 \%) ; p<0.001)$.

Conclusions Considering the relatively low cost, greater adoption of hospital-initiated tobacco cessation interventions should be considered to improve patient outcomes and decrease subsequent healthcare usage.

\section{INTRODUCTION}

Tobacco smoking remains the leading cause of premature death worldwide. ${ }^{1}$ It is unparalleled in producing early and preventable disease, disability and death, particularly related to cardiovascular and respiratory diseases and cancer. ${ }^{2}$ The magnitude of the excess morbidity, mortality and healthcare usage caused by tobacco addiction is immense. ${ }^{2} 3$ Sadly, smoking remains a widespread condition that 'presents such a mixture of lethality, prevalence and neglect despite effective and readily available interventions' ${ }^{4}$

In every jurisdiction, healthcare systems are experiencing the growing burden of chronic diseases and are under pressure to reduce 'avoidable' hospital readmissions. ${ }^{5}$ For example, the English
Department of Health introduced a policy in 2011 restricting payments of certain emergency readmissions that occur within 30 days of an initial hospital discharge. ${ }^{6}$ In the USA, the Hospital Readmissions Reductions Program was introduced as part of the Affordable Care Act; consequently, thousands of hospitals will have Medicare payments deducted by up to $3 \%$ in 2015 if patients with certain conditions (eg, myocardial infarction, heart failure, chronic obstructive pulmonary disease) return to hospital within 30 days of discharge.

In Canada, smokers average twice as many hospital days as never-daily smokers. ${ }^{8}$ Given the number of smokers admitted to hospitals, the relevance of smoking to their illness, the realities of nicotine addiction, and the challenges of withdrawal, hospitalisation presents a significant and strategic opportunity to deliver cessation interventions. ${ }^{9-11}$ There is abundant evidence that hospitalbased interventions are highly effective at helping patients quit, particularly when pharmacotherapy is provided in association with counselling and postdischarge support (risk ratio (RR), 1.37 (1.27 to 1.48); 25 trials). ${ }^{12}$ The 'Ottawa Model' for Smoking Cessation (OMSC) is a systematic approach to tobacco dependence treatment delivered within healthcare settings that has been integrated within over 120 hospitals across Canada. It involves: identifying and documenting the smoking status of all patients; providing brief counselling and inhospital pharmacotherapy to smokers; and, offering follow-up support posthospitalisation. ${ }^{13} 14$ The model has been found to improve long-term cessation by an absolute $11 \%$ (from $18 \%$ to $29 \%$; OR, 1.71 (1.11 to 2.64); $\mathrm{p}=0.02$ ) among general hospital patients. ${ }^{13}$

Evidence of the health benefits of smoking cessation is overwhelming, particularly for younger people without existing disease, but also for higher risk individuals with smoking-related illnesses. ${ }^{2}{ }^{15-17}$ A small number of investigations have assessed the health and healthcare consequences of cessation interventions delivered in hospital. They have relied on self-report, assessed only condition-specific outcomes, or used mathematical modelling to obtain outcomes. ${ }^{18-21}$

Our investigation assessed the direct effects of a hospital-initiated smoking cessation intervention (the OMSC) on mortality, rehospitalisation, emergency department (ED) use, and physician visits using healthcare administrative data. We hypothesised that, compared to controls, OMSC intervention patients would experience reduced healthcare 
usage and mortality at 30 days, 1 and 2 years following an index hospitalisation.

\section{METHODS}

\section{Design and setting}

We completed a two-group effectiveness study comparing patients who received the OMSC intervention with preimplementation controls (approved by the Ottawa Hospital Research Ethics Board: Protocol\#:2011889-01). Our study was conducted in Ontario, Canada, and expanded on a previous investigation examining smoking cessation effectiveness. ${ }^{13}$ Participants were recruited from one of 14 hospitals that, at baseline, did not have a formal cessation intervention in place but were planning to implement the OMSC. Hospital characteristics are summarised in table 1.

\section{Participants}

Patients were eligible if they: were $>17$ years of age; smoked $\geq 1$ cigarette per day in the 6 months prior to their index hospitalisation; lived in Ontario; and qualified for the Ontario Health Insurance Plan (OHIP) throughout the study. A total of 1649 patients were eligible; 741 smokers who received usual care and 908 smokers who received the OMSC (table 1).

\section{Control group}

Prior to implementing the OMSC, a consecutive sample of patients admitted to each participating hospital was screened for tobacco use by using a standardised tool. Hospitals were providing 'usual care' (typically the availability of self-help brochures) throughout this period.

\section{Intervention group}

Following control group recruitment, participating hospitals implemented the OMSC programme which involves changes in policies and procedures such that a new standard of care is introduced. ${ }^{13}{ }^{14}$ Once implemented, regardless of long-term intentions to quit, all smokers admitted to participating hospitals received the following intervention components: (1) designated healthcare staff completed a bedside consultation using a standardised form. The form documented smoking history and guided staff in recommending cessation pharmacotherapies, providing brief counselling, and enrolling patients in follow-up; (2) pharmacotherapies were ordered, as appropriate, through hospital pharmacies using preprinted order forms. Printed recommendations for continuing pharmacotherapies postdischarge were provided; (3) smokers who agreed were registered in an automated telephone follow-up system (TelASK Technologies Inc, Ottawa, Canada) at discharge and were contacted eight times over 6 months. ${ }^{22}$ Those who indicated they had relapsed to smoking or had low confidence were flagged by the system and called by smoking cessation nurse-specialists who provided counselling. After the OMSC had been in place for at least 2 months, a consecutive sample of patients who had received the OMSC was recruited using the same process and screening tool as for control group recruitment.

\section{Covariates, outcomes and data sources}

Age, sex, smoking status (yes/no to 'have you smoked any form of tobacco in the past 6 months?'), and average number of cigarettes per day were collected during patient recruitment. Additional covariates thought to potentially confound or interact with our main outcomes were gathered through data linkage and are summarised in online supplementary table S1. Participants were individually linked to administrative data bases at the Institute for Clinical Evaluative Sciences (ICES). ICES is an independent, non-profit research organisation and 'prescribed entity' under Ontario's Personal Health Information Protection Act. ${ }^{23}$

Outcomes were assessed 30 days, 1 year, and 2 years following the index hospitalisation. Our primary outcomes were allcause mortality and all-cause hospital readmission. Secondary outcomes included: smoking-related readmission; all-cause and smoking-related ED visits; and all-cause and smoking-related physician visits (specialist and general practitioner). Mortality data were acquired from the Registered Persons Database; ${ }^{24}$ hospital readmission data from the Canadian Institutes of Health Information (CIHI)-Discharge Abstract Database; ${ }^{25}$ ED visit data through CIHI's National Ambulatory Care Reporting System; ${ }^{26}$ and physician visit data through the OHIP database. We created a variable identifying whether or not events were smoking-related based on conditions in 'The Health Consequences of Smoking-50 Years of Progress: A Report of the Surgeon General'. 27

\section{Statistical methods}

Baseline characteristics were compared using $t$ tests and $\chi^{2}$ tests, as appropriate. For our principal analyses, competing-risks regression was used. ${ }^{28}$ Competing-risks regression compares the cumulative incidence of an event (ie, rehospitalisation, ED visit, physician visit) between two groups over a period of time in the presence of a competing risk (ie, death). Participants remained under observation until death or the end of the study, and those who experienced neither an event of interest nor the competing event were right-censored at the end of each analysis period. The primary independent variable in our models was group (control or intervention). Adjusted models included all covariates listed in table 2, which were treated as fixed at baseline. Hospital $(n=14)$ was used as the clustering variable. We completed a power calculation using PASS Software 12.0.3 (NCSS, LLC). Using a competing-risks model and a sample of 1400 (700 control and 700 intervention), we had $>90 \%$ power to detect a HR as modest as 0.56 , estimated from a published randomised control trial. ${ }^{18}$ An $\alpha$ level of 0.05 and two tails were used for all tests of significance. Interval estimates were based on 95\% CIs. Analyses were carried out using SAS V.9.3 (Cary, North Carolina, USA).

\section{Bias}

Several strategies were employed to ensure transparency and to address potential biases. First, an evaluation committee, including all study investigators and a third party chair, was formed prior to study commencement. The committee documented all real and potential conflicts of interest and approved the study design, outcomes, covariates, analysis plan, and dissemination plan. Once analyses were completed, the committee reviewed the study findings to verify that all plans had been followed. To reduce potential analyst bias, an initial, blinded analysis of the data was completed. A 'blinded' test data set was created by an analyst (Analyst 1) who removed identifying information (eg, dates, group assignments), randomly added observations to the smallest group in order that groups be identical in size, and randomly assigned new group numbers. The study's primary investigator (Analyst 2) and an arm's length analyst (Analyst 3) used this test data set to independently complete blinded analyses of the study's main outcomes. Results of their independent analyses were reviewed by Analyst 1, and any necessary corrections were made. Once consensus was reached (ie, statistical coding and final outputs matched), the primary analyses were repeated 
Table 1 Description of participating hospitals during patient recruitment periods

\begin{tabular}{|c|c|c|c|c|c|c|c|c|c|c|}
\hline \multirow[b]{2}{*}{ Hospital } & \multirow[b]{2}{*}{ Type } & \multirow[b]{2}{*}{ Units } & \multicolumn{4}{|c|}{ Control group recruitment } & \multicolumn{4}{|c|}{ Intervention group recruitment } \\
\hline & & & Start date & $\begin{array}{l}\text { Recruiting } \\
\text { length } \\
\text { (months) }\end{array}$ & $\begin{array}{l}\text { Total } \\
\text { patients } \\
\text { screened }\end{array}$ & $\begin{array}{l}\text { Eligible } \\
\text { smokers } \\
\text { screened }\end{array}$ & Start date & $\begin{array}{l}\text { Recruiting } \\
\text { length } \\
\text { (months) }\end{array}$ & $\begin{array}{l}\text { Total } \\
\text { patients } \\
\text { screened }\end{array}$ & $\begin{array}{l}\text { Eligible } \\
\text { smokers } \\
\text { screened }\end{array}$ \\
\hline 1 & $\mathrm{M}, \mathrm{TC}, \mathrm{T}$ & $3 / 6$ & January/2005 & 3 & 204 & 47 & July/2006 & 6 & 770 & 177 \\
\hline 2 & $\mathrm{~S}, \mathrm{G}$ & $1 / 1$ & January/2006 & 2 & 83 & 19 & February/2007 & 2 & 60 & 9 \\
\hline 3 & $\mathrm{~L}, \mathrm{TC}, \mathrm{T}$ & $2 / 14$ & January/2006 & 2 & 332 & 61 & March/2007 & 0.8 & 149 & 31 \\
\hline 4 & $\mathrm{~S}, \mathrm{G}$ & $2 / 4$ & January/2006 & 5 & 164 & 41 & May/2007 & 1 & 147 & 40 \\
\hline 5 & $S, G$ & $5 / 5$ & February/2006 & 1 & 182 & 46 & October/2007 & 2 & 161 & 37 \\
\hline 6 & $\mathrm{~L}, \mathrm{TC}, \mathrm{T}$ & $5 / 15$ & March/2006 & 2 & 481 & 114 & May/2007 & 2 & 352 & 75 \\
\hline 7 & $\mathrm{~L}, \mathrm{TC}, \mathrm{T}$ & $4 / 15$ & May/2006 & 0.3 & 504 & 98 & September/2008 & 0.8 & 852 & 129 \\
\hline 8 & $\mathrm{~S}, \mathrm{G}$ & $1 / 1$ & June/2006 & 2 & 57 & 9 & October/2007 & 8 & 122 & 28 \\
\hline 9 & $M, G$ & $6 / 6$ & June/2006 & 3 & 726 & 128 & February/2007 & 1 & 856 & 129 \\
\hline 10 & $\mathrm{~S}, \mathrm{G}$ & $2 / 3$ & June/2006 & 1 & 121 & 20 & May/2007 & 2 & 191 & 44 \\
\hline 11 & $S, G$ & $1 / 1$ & August/2006 & 1 & 104 & 29 & November/2007 & 1 & 102 & 29 \\
\hline 12 & $\mathrm{~S}, \mathrm{G}$ & $1 / 1$ & March/2007 & 1 & 26 & 4 & April/2008 & 6 & 150 & 18 \\
\hline 13 & $\mathrm{~L}, \mathrm{TC}, \mathrm{T}$ & $5 / 14$ & March/2007 & 2 & 267 & 62 & January/2008 & 4 & 357 & 82 \\
\hline \multirow[t]{2}{*}{14} & $\mathrm{M}, \mathrm{G}$ & $3 / 4$ & September/2007 & 2 & 317 & 63 & May/2009 & 1 & 348 & 80 \\
\hline & & & & & 3568 & 741 & & & 4617 & 908 \\
\hline
\end{tabular}

independently by Analysts 2 and 3 using the actual study data set to obtain final results.

The consecutive nature in which patients were recruited to the study was intended to limit selection bias, and several planned sensitivity analyses were completed to investigate potential sources of bias within the data.

\section{Sensitivity analyses}

We examined consistency of our results for 2-year mortality and all-cause readmissions by stratifying each covariate and assessing

Table 2 Patient characteristics at time of index event (smokers)

\begin{tabular}{lccc}
\hline & $\begin{array}{l}\text { Control } \\
(\mathbf{n}=641)\end{array}$ & $\begin{array}{l}\text { Intervention } \\
(\mathbf{n}=726)\end{array}$ & p Value \\
\hline Age, mean (SD) & $52.1(16.9)$ & $52.3(14.8)$ & 0.86 \\
Male sex, n (\%) & $295(46.0)$ & $367(50.5)$ & 0.09 \\
Low-SES (lowest two income & $321(50.0)$ & $357(49.1)$ & 0.68 \\
quintiles), n (\%) & & & \\
Smokes >20 cpd, n (\%) & $205(32.0)$ & $235(32.4)$ & 0.28 \\
Rurality (RIO) score, mean (SD) & $17.3(21.2)$ & $16.2(22.8)$ & 0.37 \\
High overall morbidity (RUB & $439(68.5)$ & $531(73.1)$ & 0.08 \\
score >3), n (\%) & & & \\
History of AMl, n (\%) & $80(12.5)$ & $161(22.2)$ & $<0.001$ \\
Asthma, n (\%) & $123(19.2)$ & $125(17.2)$ & 0.35 \\
History of recent cancer, n (\%) & $74(11.5)$ & $45(6.2)$ & $<0.001$ \\
CHF, n (\%) & $70(10.9)$ & $79(10.9)$ & 0.98 \\
COPD, n (\%) & $191(29.8)$ & $213(29.3)$ & 0.85 \\
Diabetes, n (\%) & $127(19.8)$ & $138(19.0)$ & 0.71 \\
Hypertension, n (\%) & $266(41.5)$ & $306(42.2)$ & 0.81 \\
History of mental illness, n (\%) & $59(9.2)$ & $90(12.4)$ & 0.06 \\
History of stroke or TIA, n (\%) & $59(9.2)$ & $48(6.6)$ & 0.07 \\
\hline
\end{tabular}

AMI, acute myocardial infarction; CHF, heart failure; COPD, chronic obstructive pulmonary disease; $C P D$, cigarettes per day; $\mathrm{N}$, number; RIO, rurality index of ontario; RUB, resource utilisation band; SES, socioeconomic status; TIA, transient ischemic attack. stratum-specific HRs. To consider the specificity of our results (ie, whether between-group differences were specific to hospitalised smokers and could be attributed to intervention exposure, rather than secular temporal trends unrelated to the intervention), we compared the 2-year probabilities of all-cause mortality and readmission between two consecutive samples of non-smoking patients who were recruited alongside the control and intervention smoker-patients. The intervention could not conceivably have influenced outcomes in the non-smokers, so any observed trends would suggest that external factors were at play. Further, we assessed whether quitting smoking improved outcomes on a subsample of smokers in both groups who consented to a 6-month follow-up call after their index hospitalisation. These participants responded yes or no to: 'Have you smoked any form of tobacco in the past 6 months?'. We combined the quitters and continued smokers from each group and continuous smoking abstinence was the main independent variable in a competing-risks regression analysis.

\section{RESULTS}

\section{Participants}

Of the 1649 eligible smokers screened, our final study dataset included 641 control (86.5\% of smokers screened) and 726 intervention smokers (80.0\% of smokers screened). A total of 282 smokers were excluded; 247 were unable to be linked (ie, unique healthcare number, name, or date of birth could not be matched); and, 35 were ineligible for OHIP for a period of time during the study. Table 2 presents baseline participant characteristics. The intervention group had a higher history of AMI $(22.2 \%$ vs $12.5 \%)$; controls had a higher history of cancer $(11.5 \%$ vs $6.2 \%)$. In all other respects, the groups were similar.

\section{Primary outcomes}

Figure 1 displays the results of our competing-risks regression analyses. The intervention group experienced significant reductions in the probabilities of all-cause readmissions, smoking-related readmissions, and all-cause ED visits at all time 

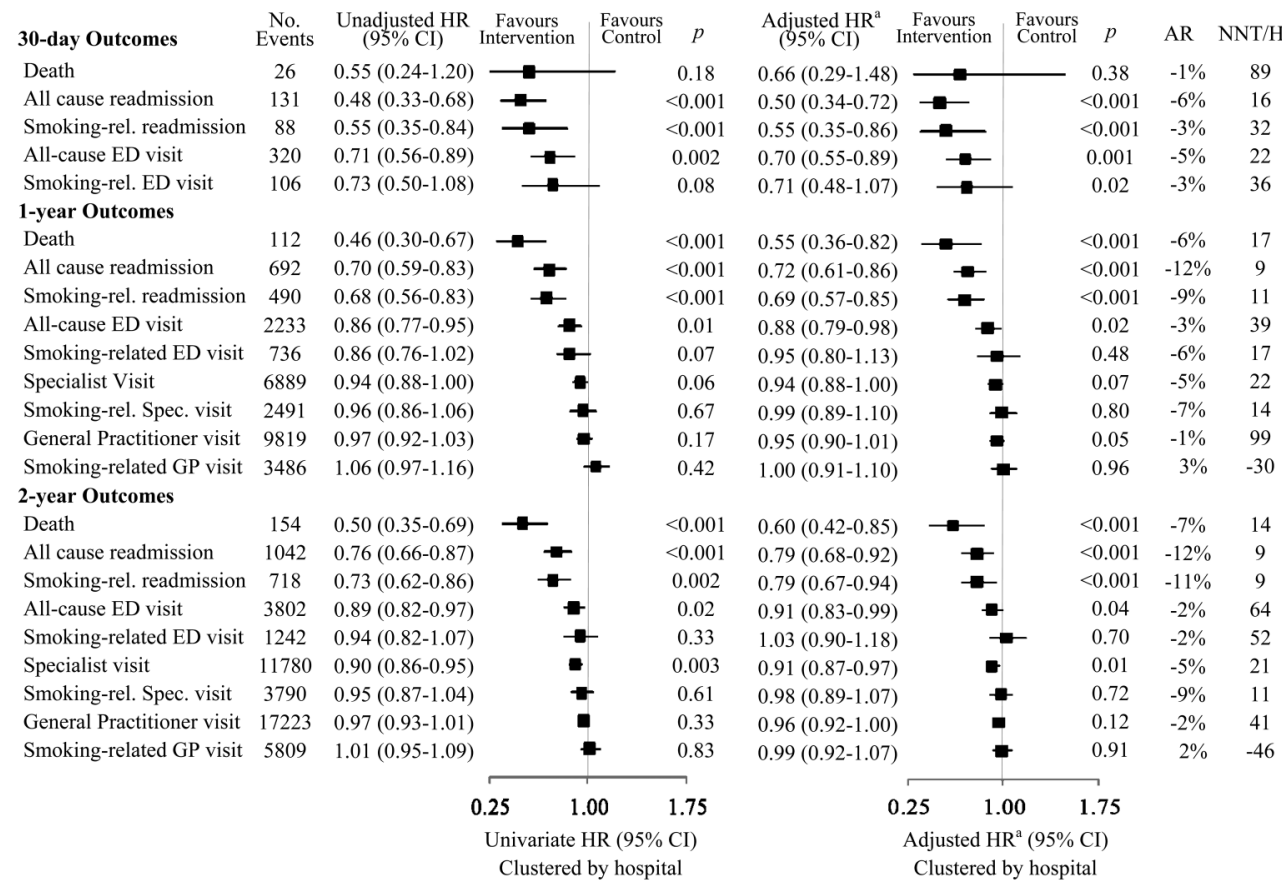

Figure 1 30-day, 1-year and 2-year outcomes of competing-risk regression for smokers receiving either OMSC (intervention, $\mathrm{n}=726$ ) or usual care (control, $\mathrm{n}=641$ ). AR, attributable risk; ED, emergency department; GP, general practitioner; NNT/H, number needed to treat/harm (negative values represent number needed to harm); Phys, physician; rel, related. A, adjusted for baseline covariates: age, sex, income, number of cigarettes smoked per day, community size, resource usage prior to index event, and history of: acute myocardial infarction, asthma, chronic obstructive pulmonary disease, heart failure, diabetes, hypertension, mental illness, stroke/transient ischemic attack.

points. The largest absolute risk reductions (ARR) were observed for all-cause readmissions at 30 days $(13.3 \%$ vs $7.1 \%$; ARR, $6.1 \%$ (2.9\% to $9.3 \%) ; \mathrm{p}<0.001)$, 1 year $(38.4 \%$ vs 26.7\%; ARR, $11.7 \%$ (6.7\% to $16.6 \%)$; $<<0.001)$, and 2 years $(45.2 \%$ vs $33.6 \%$; ARR, $11.6 \%$ (6.5\% to $16.8 \%)$; $\mathrm{p}<0.001)$. The greatest reduction in risk of all-cause ED visits was at 30 days $(20.9 \%$ vs $16.4 \%$; ARR, $4.5 \%$ (0.4\% to $8.7 \%)$; $\mathrm{p}=0.03$ ). Reduction in mortality was not evident at 30 days, but significant reductions were observed by year $1(11.4 \%$ vs $5.4 \%$; ARR $6.0 \%(3.1 \%$ to $9.0 \%) ; \mathrm{p}<0.001)$ and year $2(15.1 \%$ vs $7.9 \%$; ARR, $7.3 \%$ (3.9\% to $10.7 \%)$; $\mathrm{p}<0.001)$. Figure 2 displays the 2-year cumulative incidence curves for the primary outcomes.

\section{Sensitivity analyses}

Most stratum-specific results were consistent with our primary results, with the following exceptions. For 2-year all-cause mortality, the intervention had an impact on patients with no history of mental illness (HR; $0.68(0.58$ to 0.79$) ; \mathrm{p}<0.001)$ and those living in urban areas (HR, 0.36 (0.21 to 0.59); $\mathrm{p}<0.001$ ); however, not on those with mental illness (HR, 1.31 $(0.40$ to 5.01$) ; \mathrm{p}=0.69)$ or in rural areas (HR, $1.10(0.47$ to $2.68) ; p=0.79$ ). For 2 -year all-cause readmissions, effects differed among the following subgroups: patients with lower socioeconomic status (SES) (HR, 0.68 (0.57 to 0.80$) ; \mathrm{p}<0.001$ ) compared with higher SES (HR, 1.01 (0.78 to 1.31$) ; \mathrm{p}=0.96)$; patients with CHF (HR, 1.07 (0.78 to 1.47); $\mathrm{p}=0.42$ ) compared with patients without (HR, 0.68 (0.58 to 0.80); $\mathrm{p}<0.001)$; and, patients with mental illness (HR, 1.41 (0.96 to $2.11) ; \mathrm{p}=0.005)$ compared with those without $(\mathrm{HR}, 0.68(0.58$ to 0.79 ); $\mathrm{p}<0.001$ ).

Twelve hospitals contributed non-smoker data (non-smoker characteristics are summarised in online supplementary table
S2). The non-smokers were, on average, 12 years older than the smokers and had higher SES and overall morbidity. We observed no differences in 2-year mortality (HR, 1.14 (1.21 to 1.61); $\mathrm{p}=0.12$ ), 2-year all-cause rehospitalisation (HR, 0.99 (0.92 to $1.08) ; \mathrm{p}=0.97)$, or 2-year smoking-related rehospitalisation (HR, 1.08 (0.98 to 1.19); $\mathrm{p}=0.64)$ between 'control' and 'intervention' non-smokers.

In the subsample of smokers for whom we had 6-month smoking abstinence data, 45 of $221(20.4 \%)$ control patients had quit compared to 90 of $256(35.2 \%)$ intervention patients $\left(\chi^{2} 12.8 ; \mathrm{p}<0.001\right)$. Over 2 years, those who had been smokefree at 6 months were less likely to be rehospitalised than those who continued to smoke (HR, 0.78 (0.58 to 1.03$) ; \mathrm{p}=0.04)$, and $47 \%$ less likely to die (HR, 0.53 (0.32 to 0.83 ); $\mathrm{p}=0.07$ ).

\section{Post hoc analysis}

We explored whether the group difference in cancer history explained the observed difference in mortality. Thirty-four of 74 (45.9\%) smokers with cancer in the control group had died by year 2 , compared to 20 of $45(44.4 \%)$ in the intervention group; there was no significant difference in 2 -year probability of death between control and intervention patients with cancer (HR, 0.84 (0.43 to 1.56$) ; \mathrm{p}=0.51$ ).

\section{DISCUSSION}

Our objectives were to determine the effects of the OMSC on mortality and healthcare usage in a real-world effectiveness study with usual care controls. We observed significant reductions in 30-day, 1-year and 2-year healthcare usage, particularly for costly acute-care services, following OMSC implementation at 14 Ontario hospitals. Intervention patients experienced considerable reductions in mortality risk at 1-year and 2-year follow-ups. 

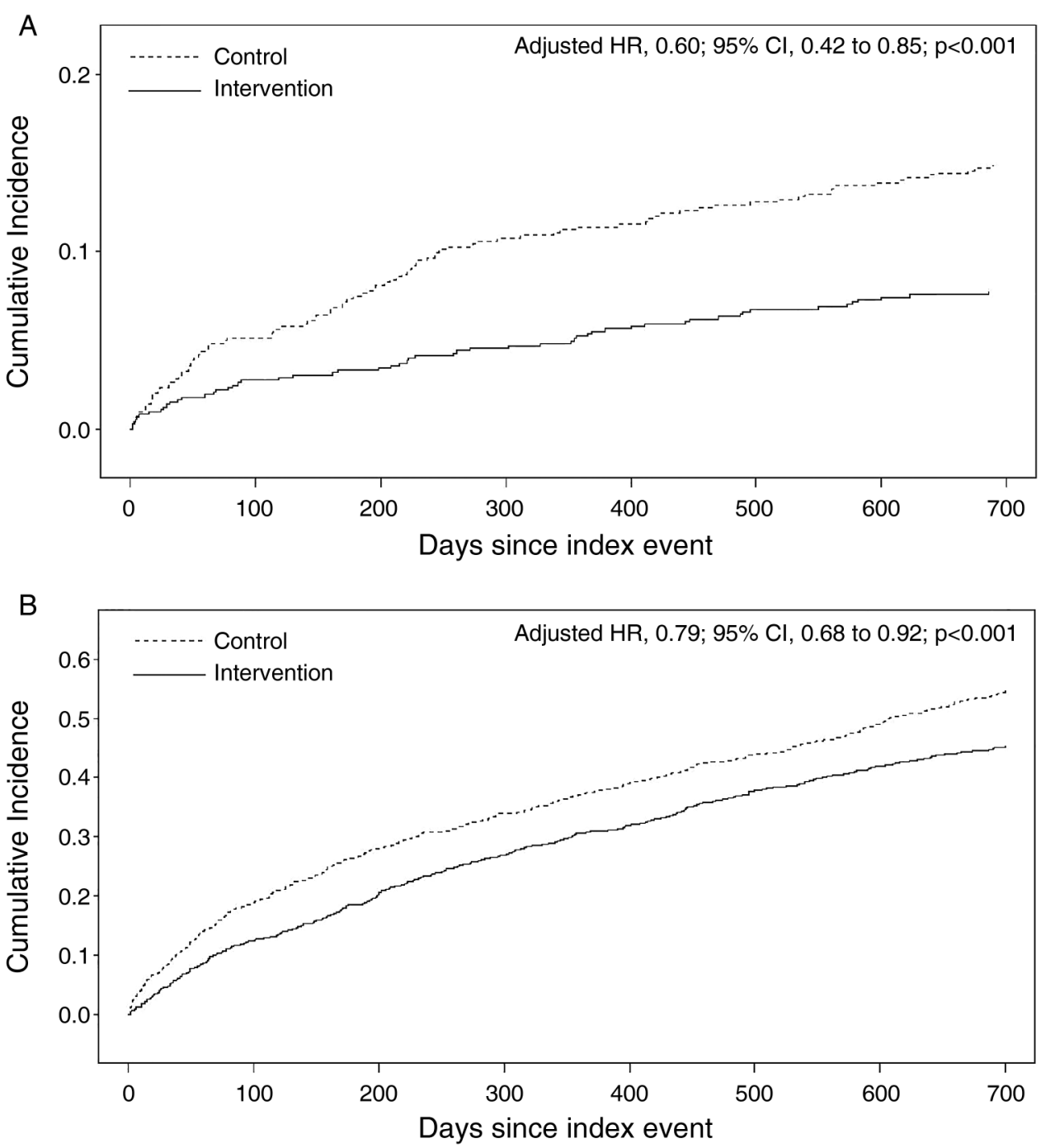

Figure 2 Cumulative incidence of mortality (Part A) and all-cause rehospitalisation (Part B) from index hospitalisation to 2-year follow-up in the control $(n=641)$ and intervention $(n=726)$ groups.

Our main findings are consistent with previous studies that have found smoking cessation to reduce morbidity and mortality in high-risk and general populations of smokers. ${ }^{15-18}{ }^{29}$ We are aware of two studies that examined rates of hospital readmission following a hospital-based cessation intervention. The first observed a $44 \%$ relative risk reduction in hospital readmissions $(16-63 \%, p=0.007)$ among smokers with acute cardiovascular conditions who were randomised to receive an intensive hospital-initiated intervention, compared to smokers in the control group. ${ }^{18}$ Our 2-year absolute risk reduction in mortality of $7.2 \%$ was similar to theirs of $9.2 \%$. The second assessed rates of psychiatric readmission following an index hospitalisation. ${ }^{20}$ Smoker-patients who received a computerised cessation intervention and offer of 6 months of nicotine replacement therapy were less likely than controls to be rehospitalised for psychiatric conditions over 18 months (OR, 1.92 (1.06 to 3.49); $\mathrm{p}=0.04)$. The above studies relied on self-reported readmission data. To our knowledge, our study is the first to compare health outcomes of smokers who received an inhospital cessation intervention to controls by directly linking to healthcare administrative data.

Our study found that attenuation of the risk of death and of hospital readmission improved during the 2-year study period; whereas, the impact on ED-visits decreased. Despite the important short-term effects of smoking abstinence, many of the effects of quitting on morbidity and mortality are observed after many years. ${ }^{17} 30$ Preventing relapse is, therefore, an important component of any cessation programme. Future hospital-initiated programmes might be enhanced by usefully examining 'opt-out' approaches to follow-up support, ${ }^{31}$ as well as relapse recovery and re-engagement strategies to support long-term abstinence. ${ }^{32}$

Our results were consistent for the majority of subgroups. The OMSC intervention was highly effective among low-SES patients. This is in contrast with a review which found that most cessation interventions do not reach, or are ineffective in, low-SES populations-populations that tend to have higher smoking rates and use a greater proportion of healthcare resources. ${ }^{33}$ Two recent randomised controlled trials (RCTs) have reported findings similar to ours. The first examined a proactive intervention delivered in primary care settings that doubled abstinence rates among low-SES smokers. ${ }^{34}$ The second evaluated an intervention that offered brief motivational counselling, 6 weeks of cost-free nicotine replacement therapy, and referral to a state quit-line to low-income smokers seen in an ED. It found that biochemically confirmed, 3-month smoking abstinence was an absolute $7.3 \%(12.2 \%$ vs $4.9 \%)$ higher among intervention group participants. ${ }^{35}$ 
We did not observe differences in primary outcomes between the 'control' and 'intervention' non-smokers suggesting that our results are specific to hospitalised smokers who received the intervention. Patients who had quit smoking experienced similar reductions in rehospitalisation and mortality as those in the intervention group, further supporting our hypotheses.

The results of our descriptive statistics revealed interesting differences between smokers and non-smokers. Smokers were hospitalised 12 years earlier than non-smokers, likely due to earlier occurrence of smoking-related illnesses. Half the smokers in our study were in the lowest two income quintiles, and $11 \%$ had a history of mental illness. By contrast, over $60 \%$ of non-smokers were in the top three income quintiles, and only $5 \%$ had a history of mental illness, confirming previously identified correlations and health disparities between and among smokers, individuals with low-SES, and those with mental illness. ${ }^{33} 3637$

\section{Limitations}

Our study included data specific to healthcare usage in Ontario; we did not have data concerning services that patients may have received in other jurisdictions, which may have resulted in an underestimate of total healthcare usage over the follow-up period. While not an RCT, we are confident in the quality of our results given the inclusion of a preimplementation control group, and that data were collected during a high-quality tobacco-use evaluation involving a consecutive sample of patients from each hospital. We did not have information about specific usage of cessation medications, nor did we have information related to exposure to other cessation services patients may have experienced. Smoking abstinence data were only available on a subsample of patients. We had no information about second-hand smoke exposure, nor did we have smoking history information for the non-smokers. Other interventions may differ from the OMSC in intensity and efficacy. Extrapolation of our results to other settings should consider these limitations.

\section{CONCLUSIONS}

Significant early and sustained reductions in mortality, rehospitalisation and ED use were observed following implementation of the OMSC hospital-based intervention. This study supports the

\section{What this paper adds}

- Little is known about the direct impacts of tobacco cessation interventions on healthcare usage. A small number of investigations have assessed the health and healthcare consequences of hospital-based cessation interventions, but until now, studies have relied on self-report or mathematical modelling to obtain health services outcomes, have assessed only disease-specific outcomes, or have not assessed outcomes both in the short ( 30 days) and long (2 years) terms.

- Our study is the first to compare the rates of mortality and downstream healthcare usage of smokers who received a hospital-based smoking cessation intervention to preintervention, 'usual care' controls using actual, healthcare administrative data. The significant short-term and long-term reductions in mortality, rehospitalisation, and emergency department visits experienced by intervention participants are of substantial value, not only for patients, but for clinicians and healthcare payers looking for strategies to manage the growing burden of chronic diseases. case for broader adoption of systematic cessation interventions within hospitals that advise patients about quitting, initiate cessation pharmacotherapies, and attach patients to follow-up support posthospitalisation. Such strategies, we would argue, will reduce subsequent healthcare consumption and the burden of the 'revolving door' patient; but, most importantly, distinctly enhance the well-being of patients.

Acknowledgements The authors would like to thank Bonnie Quinlan, Debbie Aitken, Lynne Robert and Louise Leger-Caldwell for their clinical expertise and contributions. They gratefully acknowledge Beverley Bean, Christine Blais, Helen Brezynskie, Elizabeth Contestabile, Donna Cousineau, Marcia Foster, Deirdre Gilbert, Sophia Gocan, Heather Houlahan, Lyse Huneault, Natalie Ladouceur, Jan Leahy, Carlene MacDonald, Maureen Magill, Chris McLaughlin, Toba Miller, Kelly Mitten, Liane Murphy, Helen Ostap, Stephanie Pick, Laurie Scissons, Karen Scobie, Jennifer Smylie, Lisa Whalen and the other participating hospital coordinators and managers. They wish to extend their thanks to Ashley Armstrong, Rebecca Nadler, Shaughna McDonald, Angela Raymond, Nelson Chong, Jenna Wong and Heather Manson for their assistance with the investigations.

Disclaimer The opinions, results and conclusions included in this report are those of the authors and are independent from the funding sources. No endorsement by the Institute for Clinical Evaluative Sciences (ICES) or the Ontario Ministry of Health and Long-Term Care (MOHLTC) is intended or should be inferred.

Contributors KAM, RDR and DGM conceived the study. KAM proposed the design and methodology, which were approved by DGM, SJH, ALP, DC, GAW and RDR. KAM drafted the analysis plan, which was reviewed and approved by SJH. LAH assisted in data preparation. KAM and JY completed the blinded and primary data analyses. KAM completed all other data analyses and wrote the manuscript. All authors contributed to the review and editing of the final manuscript. KAM had full access to all the data in the study, and had final responsibility for the decision to submit for publication.

Funding KAM was supported by a 2-year fellowship (2009-2011) in Population Intervention for Chronic Disease Prevention from the Canadian Institutes of Health Research. Additional financial support was provided through a contribution agreement between the University of Ottawa Heart Institute and the Ontario Ministry of Health and Long Term Care (Smoke Free Ontario Strategy). The funders did not contribute to any of the following: design and conduct of the study; collection, management, analysis and interpretation of the data; and preparation, review, or approval of the manuscript.

Competing interests KAM, ALP and RDR are inventors of the Ottawa Model for Smoking Cessation, a registered trademark of the University of Ottawa Heart Institute. ALP has received payment for consultancy from Pfizer Inc., Johnson \& Johnson and Glaxo Smith Klein, and for lectures from Johnson \& Johnson and Pfizer Inc. He has received research funding from Pfizer Inc. RDR has received payment for lectures from Johnson \& Johnson and Pfizer Inc. and is currently holding a research grant through the Pfizer Global Research Awards for Nicotine Dependence competition. The other authors declare no conflicts of interest.

Ethics approval Ottawa Hospital Research Ethics Board.

Provenance and peer review Not commissioned; externally peer reviewed.

Data sharing statement Data sharing will be considered upon request made to the corresponding author.

Open Access This is an Open Access article distributed in accordance with the Creative Commons Attribution Non Commercial (CC BY-NC 4.0) license, which permits others to distribute, remix, adapt, build upon this work non-commercially, and license their derivative works on different terms, provided the original work is properly cited and the use is non-commercial. See: http://creativecommons.org/ licenses/by-nc/4.0/

\section{REFERENCES}

1 World Health Organization. WHO Report on the global tobacco epidemic, 2013 Enforcing bans on tobacco advertising, promotion and sponsorship. Geneva: 2013. http://www.who.int/tobacco/global_report/2013/en/ (accessed Aug 2014).

2 The Health Consequences of Smoking: 50 Years of Progress. A Report of the Surgeon General. Atlanta: Department of Health and Human Services, Centers for Disease Control and Prevention, National Center for Chronic Disease Prevention and Health Promotion, Office on Smoking and Health, 2014. http://www. surgeongeneral.gov/library/reports/50-years-of-progress/front-matter.pdf (accessed 12 Jul 2014).

3 Carter BD, Abnet CC, Feskanich D, et al. Smoking and mortality-beyond established causes. N Engl J Med 2015;372:631-40.

4 Fiore MC, Jaen CR, Baker TB, et al. Treating tobacco use and dependence: 2008 update. Rockville, MD, USA: Department of Health and Human Services, 2008:12. 
5 Abegunde DO, Mathers CD, Adam T, et al. The burden and costs of chronic diseases in low-income and middle-income countries. Lancet 2007;370:1929-38.

6 Kristensen SR, Bech M, Quentin W. A roadmap for comparing readmission policies with application to Denmark, England, Germany and the United States. Health Policy 2015;119:264-73.

7 Readmissions Reduction Program. Baltimore: Centers for Medicare \& Medicaid Services, 2014. http://cms.gov/Medicare/Medicare-Fee-for-Service-Payment/ AcutelnpatientPPS/Readmissions-Reduction-Program.html (accessed 9 Dec 2014).

8 Wilkins K, Shields M, Rotermann M. Smokers' use of acute care hospitals—A prospective study. Health Rep 2009;20:1-9.

9 Benowitz NL. Nicotine Addiction. N Engl J Med 2010;362:2295-303.

10 Pfuntner A, Wier LM, Stocks C. Most Frequent Conditions in U.S. Hospitals, 2010. Agency for Healthcare Research and Quality, 2013. http://www.hcup-us.ahrq.gov/ reports/statbriefs/sb148.pdf (accessed 17 Nov 2014).

11 Rigotti NA, Regan S, Levy DE, et al. Sustained care intervention and postdischarge smoking cessation among hospitalized adults: a randomized clinical trial. JAMA 2014:312:719-28.

12 Rigotti NA, Clair C, Munafò MR, et al. Interventions for smoking cessation in hospitalised patients. Cochrane Database Syst Rev 2012:5:CD001837.

13 Reid RD, Mullen KA, Slovinec D'Angelo ME, et al. Smoking cessation for hospitalized smokers: an evaluation of the "Ottawa Model". Nicotine Tob Res 2010;12:11-18.

14 Reid RD, Pipe AL, Quinlan B. Promoting smoking cessation during hospitalization for coronary artery disease. Can I Cardiol 2006;22:775-80.

15 Bullen C. Impact of tobacco smoking and smoking cessation on cardiovascular risk and disease. Expert Rev Cardiovasc Ther 2008:6:883-95.

16 Godtfredsen NS, Lam TH, Hansel TT, et al. COPD-related morbidity and mortality after smoking cessation: status of the evidence. Eur Respir J 2008:32:844-53.

17 Jha P, Ramasundarahettige C, Landsman V, et al. 21st-century hazards of smoking and benefits of cessation in the United States. N Engl I Med 2013:368:341-50.

18 Mohiuddin SM, Mooss AN, Hunter CB, et al. Intensive smoking cessation intervention reduces mortality in high-risk smokers with cardiovascular disease. Chest 2007;131:446-52.

19 Mullen KA, Coyle D, Manuel D, et al. Economic evaluation of a hospital-initiated intervention for smokers with chronic disease, in Ontario, Canada. Tob Control 2015;24:489-96.

20 Prochaska JJ, Hall SE, Delucchi K, et al. Efficacy of initiating tobacco dependence treatment in inpatient psychiatry: a randomized controlled trial. Am J Public Health 2014;104:1557-65

21 Van Spall HG, Chong A, Tu JV. Inpatient smoking-cessation counseling and all-cause mortality in patients with acute myocardial infarction. Am Heart $J$ 2007:154:213-20.
22 Reid RD, Pipe AL, Quinlan B, et al. Interactive voice response telephony to promote smoking cessation in patients with heart disease: a pilot study. Patient Educ Couns 2007;66:319-26

23 Personal Health Information Protection Act, 2004. Toronto: Ontario Ministry of Health and Long Term Care, 2010. http://www.e-laws.gov.on.ca/html/statutes/ english/elaws_statutes_04p03_e.htm (accessed Nov 2014).

24 Registered Persons Database. Ontario Ministry of Health and Long Term Care, 2011. http://www.health.gov.on.ca/ (accessed Jul 2012).

25 DAD Abstracting Manuel. Ottawa: Canadian Institute for Health Information, 2009. http://www.cihi.ca/CIHI-ext-portal/pdf/internet/dad_data_elements_2009_2010_en (accessed 10 Jul 2012).

26 NACRS Data Elements 2010-2011. Ottawa: Canadian Institute for Health Information, 2010. http://www.cihi.ca/CIHI-ext-portal/pdf/internet/nacrs_data_ element_table_en (accessed Aug 2012).

27 Resource Manual for Physicians. Kingston: Ontario Ministry of Health and Long Term Care, Health Services Branch, 2014. http://www.health.gov.on.ca/english/ providers/pub/ohip/physmanual/download/resource_manual_phys_march2014_en. pdf (accessed Jul 2012).

28 Fine JP, Gray RJ. A proportional hazards model for the subdistribution of a competing risk. J Am Statist Assoc 1999;94:496-509.

29 Parsons A, Daley A, Begh R, et al. Influence of smoking cessation after diagnosis of early stage lung cancer on prognosis: systematic review of observational studies with meta-analysis. BMJ 2010;340:b5569.

30 Hoogenveen RT, van Baal PH, Boshuizen $\mathrm{HC}$, et al. Dynamic effects of smoking cessation on disease incidence, mortality and quality of life: The role of time since cessation. Cost Eff Resour Alloc 2008;6:1-15.

31 Richter KP, Ellerbeck EF. It's time to change the default for tobacco treatment. Addiction 2015;110:381-6.

32 Schlam TR, Baker TB. Interventions for tobacco smoking. Annu Rev Clin Psychol 2013;9:675-702

33 Hiscock R, Bauld L, Amos A, et al. Socioeconomic status and smoking: a review. Ann N Y Acad Sci 2012;1248:107-23.

34 Haas JS, Linder JA, Park ER, et al. Proactive tobacco cessation outreach to smokers of low socioeconomic status: A randomized clinical trial. JAMA Intern Med 2015:175:218-26.

35 Bernstein SL, D'Onofrio G, Rosner J, et al. Successful Tobacco Dependence Treatment in Low-Income Emergency Department Patients: A Randomized Trial. Ann Emerg Med 2015;66:140-7.

36 Gilbody S, Peckham E, Man MS, et al. Bespoke smoking cessation for people with severe mental ill health (SCIMITAR): a pilot randomised controlled trial. Lancet Psychiatry 2015:2:395-402.

37 Lawrence D, Mitrou F, Zubrick SR. Smoking and mental illness: results from population surveys in Australia and the United States. BMC Public Health 2009:9:285. 